\title{
A Schwarz lemma on complex ellipsoids
}

\author{
by HideTAKa Hamada (Kitakyushu)
}

Abstract. We give a Schwarz lemma on complex ellipsoids.

1. Introduction. Let $\Delta=\{z \in \mathbb{C}|| z \mid<1\}$ be the unit disc in $\mathbb{C}$. The Schwarz lemma in one complex variable is as follows.

THEOREM 1. (i) Let $f: \Delta \rightarrow \Delta$ be a holomorphic map such that $f(0)=$ 0. Then $|f(z)| \leq|z|$ for all $z \in \Delta$.

(ii) If, moreover, there exists $z_{0} \in \Delta \backslash\{0\}$ such that $\left|f\left(z_{0}\right)\right|=\left|z_{0}\right|$, or if $\left|f^{\prime}(0)\right|=1$, then there exists a complex number $\lambda$ of absolute value 1 such that $f(z)=\lambda z$ and $f$ is an automorphism of $\Delta$.

Let $D$ be the unit ball in $\mathbb{C}^{n}$ for some norm $\|\cdot\|$, and let $f: D \rightarrow D$ be a holomorphic map such that $f(0)=0$. By the Hahn-Banach theorem, we have $\|f(z)\| \leq\|z\|$ for all $z \in D$. As a generalization of part (ii) of the above theorem, Vigué [7] proved the following.

TheOREM 2. Let $D$ be the unit ball in $\mathbb{C}^{n}$ for some norm $\|\cdot\|$, and let $f: D \rightarrow D$ be a holomorphic map such that $f(0)=0$. Assume that every boundary point of $D$ is a complex extreme point of $\bar{D}$. If one of the following conditions is satisfied, then $f$ is a linear automorphism of $\mathbb{C}^{n}$.

$\left(\mathrm{H}_{1}\right)$ There exists a nonempty open subset $U$ of $D$ such that $\|f(x)\|=$ $\|x\|$ on $U$.

$\left(\mathrm{H}_{2}\right)$ There exists a nonempty open subset $U$ of $D$ such that $c_{D}(f(0)$, $f(x))=c_{D}(0, x)$ on $U$, where $c_{D}$ denotes the Carathéodory distance on $D$.

$\left(\mathrm{H}_{3}\right)$ There exists a nonempty open subset $V$ of $T_{0}(D)$ such that $E_{D}\left(f(0), f^{\prime}(0) v\right)=E_{D}(0, v)$ on $V$, where $E_{D}$ denotes the infinitesimal Carathéodory metric on $D$.

1991 Mathematics Subject Classification: Primary 32A10.

Key words and phrases: Schwarz lemma, complex ellipsoid, extreme point, balanced domain, Minkowski function, geodesics. 
Moreover, he showed that if there exists a point $a \in U \backslash\{0\}$ such that $f(a)=a$, or if the boundary $\partial D$ of $D$ is a real-analytic submanifold of $\mathbb{C}^{n}$, then $f$ is a linear automorphism of $D$. As a corollary, he proved that if $D$ is the unit ball of $\mathbb{C}^{n}$ for the Euclidean norm on $\mathbb{C}^{n}$, then $f$ is a linear automorphism of $D$. But, in the above results, the conditions $\left(\mathrm{H}_{1}\right)$ and $\left(\mathrm{H}_{2}\right)$ are strong, because a point in $\Delta$ is of codimension 1 and an open set in $D$ is of codimension 0 . The author [2] announced that Vigué's results hold under the hypothesis that one of the conditions $\left(\mathrm{H}_{1}\right),\left(\mathrm{H}_{2}\right)$ is satisfied for some local complex submanifold of codimension 1 instead of an open subset.

The aim of the present paper is to consider an analogous result on complex ellipsoids $\mathcal{E}(p)$. However, $\mathcal{E}(p)$ is not convex in general. For a bounded balanced convex domain $D$, the Minkowski function $h$ of $D$ is a norm on $\mathbb{C}^{n}$ and $D$ is the unit ball in $\mathbb{C}^{n}$ with respect to this norm. Also, $c_{D}=\widetilde{k}_{D}$ and $E_{D}=\kappa_{D}$ in the convex case (Lempert [4], [5], Royden-Wong [6]), where $\widetilde{k}_{D}$ is the Lempert function and $\kappa_{D}$ is the the Kobayashi-Royden pseudometric for $D$. So we use $h, \widetilde{k}_{D}$ and $\kappa_{D}$ instead of $\|\cdot\|, c_{D}$ and $E_{D}$. First we give a theorem on some bounded balanced pseudoconvex domains which corresponds to Theorem 2. Then we show that if $D=\mathcal{E}(p)$, then $f$ is a linear automorphism of $\mathcal{E}(p)$. We also give an example showing that our hypothesis cannot be weakened.

Some ideas of this paper come from Dini-Primicerio [1] and Vigué [7], [8].

2. Main results. The Lempert function $\widetilde{k}_{D}$ and the Kobayashi-Royden pseudometric $\kappa_{D}$ for a domain $D$ in $\mathbb{C}^{n}$ are defined as follows:

$$
\begin{gathered}
\widetilde{k}_{D}(x, y)=\inf \{\varrho(\xi, \eta) \mid \xi, \eta \in \Delta, \quad \exists \varphi \in H(\Delta, D) \text { such that } \\
\varphi(\xi)=x, \varphi(\eta)=y\}, \\
\kappa_{D}(z ; X)=\inf \{\gamma(\lambda)|\alpha| \mid \exists \varphi \in H(\Delta, D), \quad \exists \lambda \in \Delta \text { such that } \\
\left.\varphi(\lambda)=z, \alpha \varphi^{\prime}(\lambda)=X\right\},
\end{gathered}
$$

where $\varrho$ is the Poincaré distance on the unit disc $\Delta$ and $\gamma(\lambda)=1 /\left(1-|\lambda|^{2}\right)$.

Let $D$ be a balanced pseudoconvex domain with Minkowski function $h$ in $\mathbb{C}^{n}$. Then we have (Propositions 3.1.10 and 3.5.3 of Jarnicki and Pflug [3])

$$
\begin{aligned}
\widetilde{k}_{D}(0, x) & =\varrho(0, h(x)) & & \text { for any } x \text { in } D, \\
\kappa_{D}(0, X) & =h(X) & & \text { for any } X \text { in } \mathbb{C}^{n} .
\end{aligned}
$$

Let $f$ be a holomorphic map from $D$ to $D$ such that $f(0)=0$. By (1) and the distance decreasing property of the Lempert functions, we have

$$
\varrho(0, h(z))=\widetilde{k}_{D}(0, z) \geq \widetilde{k}_{D}(0, f(z))=\varrho(0, h(f(z))) .
$$

Since $\varrho(0, r)$ is increasing for $0 \leq r<1$, we obtain $h(f(z)) \leq h(z)$. This is a generalization of part (i) of the Schwarz lemma to balanced pseudoconvex domains. 
A boundary point $x$ of $D$ is said to be an extreme point of $\bar{D}$ if there is no non-constant holomorphic mapping $g: \Delta \rightarrow \bar{D}$ with $x=g(0)$. For example, $C^{2}$-smooth strictly pseudoconvex boundary points are extreme points (p. 257 of Jarnicki and Pflug [3]).

A mapping $\varphi \in H(\Delta, D)$ is said to be a complex $\widetilde{k}_{D}$-geodesic for $(x, y)$ if there exist points $\xi, \eta \in \Delta$ such that $\varphi(\xi)=x, \varphi(\eta)=y$, and $\widetilde{k}_{D}(x, y)=$ $\varrho(\xi, \eta)$.

A mapping $\varphi \in H(\Delta, D)$ is said to be a complex $\kappa_{D^{-}}$geodesic for $(z, X)$ if there exist $\lambda \in \Delta$ and $\alpha \in \mathbb{C}$ such that $\varphi(\lambda)=z, \alpha \varphi^{\prime}(\lambda)=X$, and $\kappa_{D}(z, X)=\gamma(\lambda)|\alpha|$.

Using (1), (2) and complex $\widetilde{k}_{D}$-geodesics or $\kappa_{D}$-geodesics, we have the following proposition (cf. Vigué [7], [8], Hamada [2]).

Proposition 1. Let $D_{j}$ be bounded balanced pseudoconvex domains with Minkowski functions $h_{j}$ in $\mathbb{C}^{n_{j}}$ for $j=1,2$, and let $f: D_{1} \rightarrow D_{2}$ be a holomorphic map such that $f(0)=0$. Let $f(z)=\sum_{m=1}^{\infty} P_{m}(z)$ be the development of $f$ in vector-valued homogeneous polynomials $P_{m}$ in a neighborhood of 0 , where $\operatorname{deg} P_{m}=m$ for each $m$. Let $x \in D_{1} \backslash\{0\}$. If one of the following conditions is satisfied, then we have $P_{m}(x)=0$ for $m \geq 2$.

$\left(\mathrm{H}_{1}^{\prime}\right) h_{2}(f(x))=h_{1}(x)$ and $f(x) / h_{2}(f(x))$ is an extreme point of $\bar{D}_{2}$.

$\left(\mathrm{H}_{2}^{\prime}\right) \widetilde{k}_{D_{2}}(f(0), f(x))=\widetilde{k}_{D_{1}}(0, x)$ and $f(x) / h_{2}(f(x))$ is an extreme point of $\bar{D}_{2}$.

$\left(\mathrm{H}_{3}^{\prime}\right) \kappa_{D_{2}}\left(f(0), f^{\prime}(0) x\right)=\kappa_{D_{1}}(0, x)$ and $f^{\prime}(0) x / h_{2}\left(f^{\prime}(0) x\right)$ is an extreme point of $\bar{D}_{2}$.

Proof. By $(1)$, the conditions $\left(\mathrm{H}_{1}^{\prime}\right)$ and $\left(\mathrm{H}_{2}^{\prime}\right)$ are equivalent. Let

$$
\varphi(\zeta)=\zeta \frac{x}{h_{1}(x)}
$$

Then $\varphi$ is a complex $\widetilde{k}_{D_{1}}$-geodesic and $\kappa_{D_{1}}$-geodesic for $(0, x)$. Suppose that $\left(\mathrm{H}_{1}^{\prime}\right)$ or $\left(\mathrm{H}_{2}^{\prime}\right)$ is satisfied. Since

$$
\widetilde{k}_{D_{2}}\left(f \circ \varphi(0), f \circ \varphi\left(h_{1}(x)\right)\right)=\widetilde{k}_{D_{2}}(0, f(x))=\widetilde{k}_{D_{1}}(0, x)=\varrho\left(0, h_{1}(x)\right),
$$

$f \circ \varphi$ is a complex $\widetilde{k}_{D_{2}}$-geodesic for $(0, f(x))$. By Proposition 8.3.5(a) of Jarnicki and Pflug [3],

Since

$$
f \circ \varphi(\zeta)=\zeta \frac{f(x)}{h_{2}(f(x))}
$$

$$
f \circ \varphi(\zeta)=\sum P_{m}\left(\zeta \frac{x}{h_{1}(x)}\right)=\sum\left(\frac{\zeta}{h_{1}(x)}\right)^{m} P_{m}(x)
$$

in a neighborhood of $0, P_{m}(x)=0$ for $m \geq 2$. 
Suppose that $\left(\mathrm{H}_{3}^{\prime}\right)$ is satisfied. Since

$\kappa_{D_{2}}\left(0, f^{\prime}(0) x\right)=\kappa_{D_{1}}(0, x)=h_{1}(x) \quad$ and $\quad h_{1}(x)(f \circ \varphi)^{\prime}(0)=f^{\prime}(0) x$,

$f \circ \varphi$ is a complex $\kappa_{D_{2}}$-geodesic for $\left(0, f^{\prime}(0) x\right)$. By Proposition 8.3.5(a) of Jarnicki and Pflug [3],

$$
f \circ \varphi(\zeta)=\zeta e^{i \theta} \frac{f^{\prime}(0) x}{h_{2}\left(f^{\prime}(0) x\right)}
$$

for some $\theta \in \mathbb{R}$. The rest of the argument is the same as above. This completes the proof.

The following proposition is a key for proving our theorem (Hamada [2]).

Proposition 2. Let $U$ be an open subset of $\mathbb{C}^{n}$. Let $M$ be a complex submanifold of $U$ of dimension $n-1$. Assume that there exists a point a in $M$ such that $a+T_{a}(M)$ does not contain the origin. Then there exists a neighborhood $U_{1}$ of a in $\mathbb{C}^{n}$ such that $U_{1} \subset \mathbb{C} M=\{t x \mid t \in \mathbb{C}, x \in M\}$.

Proof. To prove this proposition, it is enough to prove the following claim.

Claim. For any $x$ in $M$, let $g(x)$ be the intersection point of $a+T_{a}(M)$ and the complex line through $x$ and the origin $O$. Then $g$ is a biholomorphic map from a neighborhood $W_{M}$ of a in $M$ onto a neighborhood $W_{T}$ of a in $a+T_{a}(M)$.

Assume the claim is proved. Since there exists an open neighborhood $U_{1}$ of $a$ in $\mathbb{C}^{n}$ such that $U_{1} \subset \mathbb{C} W_{T}$, we obtain $U_{1} \subset \mathbb{C} M$.

Now we will prove the claim. By an affine coordinate change, we may assume that $a=0, M=\left\{z_{n}=\psi\left(z^{\prime}\right)\right\}$ with $\psi(0)=0, d \psi(0)=0$, where $\left(z^{\prime}, z_{n}\right) \in \mathbb{C}^{n}$. Then $\left(z^{\prime}, \psi\left(z^{\prime}\right)\right)$ gives a local parametrization of $M$ at $a$, $a+T_{a}(M)=\left\{z_{n}=0\right\}$ and $O=\left(b_{1}, \ldots, b_{n}\right)$ with $b_{n} \neq 0$. Let $g\left(z^{\prime}, \psi\left(z^{\prime}\right)\right)=$ $\left(g_{1}\left(z^{\prime}\right), \ldots, g_{n-1}\left(z^{\prime}\right), 0\right)$. Since

$$
g_{i}\left(z^{\prime}, \psi\left(z^{\prime}\right)\right)=b_{i}+\frac{b_{n}}{b_{n}-\psi\left(z^{\prime}\right)}\left(z_{i}-b_{i}\right)
$$

for sufficiently small $z^{\prime}$, we have

$$
\frac{\partial g_{i}}{\partial z_{j}}(0)=\delta_{i j} \quad(1 \leq i, j \leq n-1) .
$$

Therefore $g$ is biholomorphic in a neighborhood $W_{M}$ of $a$. This completes the proof.

From now on, we assume that $D$ is a bounded balanced pseudoconvex domain in $\mathbb{C}^{n}$ which satisfies the following condition:

$(*)$ For any $1 \leq j_{1}<\ldots<j_{k} \leq n(0 \leq k \leq n-1)$, let

$$
\widetilde{D}=D \cap\left\{z_{j_{1}}=\ldots=z_{j_{k}}=0\right\}
$$


be a domain in $\mathbb{C}^{n-k}$. Then every point of $\partial \widetilde{D} \cap\left(\mathbb{C}^{*}\right)^{n-k}$ is an extreme point of $\overline{\widetilde{D}}$.

By the above two propositions, we have the following theorem.

THEOREM 3. Let $D$ be a bounded balanced pseudoconvex domain with Minkowski function $h$ in $\mathbb{C}^{n}$ which satisfies the condition $(*)$, and let $f$ : $D \rightarrow D$ be a holomorphic map such that $f(0)=0$. Let $M$ be a connected complex submanifold of dimension $n-1$ of an open subset $U$ of $D$ such that $a+T_{a}(M)$ does not contain the origin for some $a$ in $M$. Let $V$ be a connected open subset of $T_{0}(D)$. If one of the following conditions is satisfied, then $f$ is a linear automorphism of $\mathbb{C}^{n}$.

$\left(\mathrm{H}_{1}^{\prime \prime}\right) h(f(x))=h(x)$ on $M$.

$\left(\mathrm{H}_{2}^{\prime \prime}\right) \widetilde{k}_{D}(f(0), f(x))=\widetilde{k}_{D}(0, x)$ on $M$.

$\left(\mathrm{H}_{3}^{\prime \prime}\right) \kappa_{D}\left(f(0), f^{\prime}(0) v\right)=\kappa_{D}(0, v)$ on $V$.

Proof. Suppose that $\left(\mathrm{H}_{1}^{\prime \prime}\right)$ or $\left(\mathrm{H}_{2}^{\prime \prime}\right)$ is satisfied. We may assume that for any $a \in M, a+T_{a}(M)$ does not contain the origin, the functions $f_{1}, \ldots, f_{k}$ do not vanish on $M$ and the functions $f_{k+1}, \ldots, f_{n}$ are identically 0 on $M$ for some $k, 1 \leq k \leq n$. Let

$$
\widetilde{D}=D \cap\left\{z_{k+1}=\ldots=z_{n}=0\right\} \quad \text { and } \quad \widetilde{f}=\left(f_{1}, \ldots, f_{k}\right) .
$$

Then $\widetilde{D}$ is a bounded balanced pseudoconvex domain in $\mathbb{C}^{k}$ with Minkowski function $\widetilde{h}=h \mid \widetilde{D}$, and $\widetilde{f}$ is a holomorphic map from $D$ to $\widetilde{D}$ with $\widetilde{f}(0)$ $=0$. Since the functions $f_{1}, \ldots, f_{k}$ do not vanish on $M, \widetilde{f}(x) / \widetilde{h}(\widetilde{f}(x))$ is an extreme point of $\overline{\widetilde{D}}$ for any $x \in M$. Let

$$
\tilde{f}(z)=\sum_{m=1}^{\infty} P_{m}(z)
$$

be the development of $\tilde{f}$ in vector-valued homogeneous polynomials $P_{m}$ in a neighborhood of 0 , where $\operatorname{deg} P_{m}=m$ for each $m$. Since $\widetilde{h}(\widetilde{f}(x))=$ $h(f(x))=h(x)$ on $M$, we have $P_{m}(x)=0$ on a nonempty open subset $U_{1}$ of $D$ for $m \geq 2$ by Propositions 1 and 2. By the analytic continuation theorem, $P_{m}$ is identically 0 for $m \geq 2$. Therefore $\widetilde{f}$ is linear. By Proposition 2, we have $\widetilde{h}(\widetilde{f}(x))=h(x)$ on $U_{1}$. We can show that $\operatorname{Ker}(\widetilde{f})=0$ as in Vigué [7]. Then $k$ must be $n$ and $f$ is a linear automorphism of $\mathbb{C}^{n}$.

Suppose that $\left(\mathrm{H}_{3}^{\prime \prime}\right)$ is satisfied. We may assume that $\partial f_{1}(0), \ldots, \partial f_{k}(0)$ are not 0 and $\partial f_{k+1}(0), \ldots, \partial f_{n}(0)$ are 0 for some $k, 1 \leq k \leq n$. Let

$$
\widetilde{D}=D \cap\left\{z_{k+1}=\ldots=z_{n}=0\right\} \quad \text { and } \quad \widetilde{f}=\left(f_{1}, \ldots, f_{k}\right) .
$$

Then $\widetilde{D}$ is a bounded balanced pseudoconvex domain in $\mathbb{C}^{k}$ with Minkowski function $\widetilde{h}=h \mid \widetilde{D}$, and $\widetilde{f}$ is a holomorphic map from $D$ to $\widetilde{D}$ with $\widetilde{f}(0)=0$. 
We may assume that $\partial f_{1}(0) \cdot\left(\sum v_{j} \partial / \partial z_{j}\right), \ldots, \partial f_{k}(0) \cdot\left(\sum v_{j} \partial / \partial z_{j}\right)$ do not vanish for any $v \in V$. Then $\widetilde{f}^{\prime}(0) v / \widetilde{h}\left(\widetilde{f}^{\prime}(0) v\right)$ is an extreme point of $\widetilde{D}$ for any $v \in V$. The rest of the argument is the same as above. This completes the proof.

For $p=\left(p_{1}, \ldots, p_{n}\right)$ with $p_{1}, \ldots, p_{n}>0$, let

$$
\mathcal{E}(p)=\left\{\left.\left(z_{1}, \ldots, z_{n}\right)\left|\sum_{j=1}^{n}\right| z_{j}\right|^{2 p_{j}}<1\right\} .
$$

Then $\mathcal{E}(p)$ is a bounded balanced pseudoconvex domain which satisfies the condition (*) (cf. p. 264 of Jarnicki and Pflug [3]). Let $f$ be a holomorphic map from $\mathcal{E}(p)$ to itself which satisfies the condition of Theorem 3. Then $f$ is a linear automorphism of $\mathbb{C}^{n}$ by Theorem 3. Moreover, we can show that $f$ is a linear automorphism of $\mathcal{E}(p)$ using the idea of Dini and Primicerio [1].

THEOREM 4. Let $f$ be a holomorphic map from $\mathcal{E}(p)$ to itself such that $f(0)=0$. Let $M$ be a connected complex submanifold of dimension $n-1$ of an open subset $U$ of $\mathcal{E}(p)$ such that $a+T_{a}(M)$ does not contain the origin for some a in $M$. Let $V$ be a connected open subset of $T_{0}(D)$. If one of the following conditions is satisfied, then $f$ is a linear automorphism of $\mathcal{E}(p)$.

$\left(\mathrm{H}_{1}^{\prime \prime}\right) h(f(x))=h(x)$ on $M$, where $h$ is the Minkowski function of $\mathcal{E}(p)$.

$\left(\mathrm{H}_{2}^{\prime \prime}\right) \widetilde{k}_{\mathcal{E}(p)}(f(0), f(x))=\widetilde{k}_{\mathcal{E}(p)}(0, x)$ on $M$.

$\left(\mathrm{H}_{3}^{\prime \prime}\right) \kappa_{\mathcal{E}(p)}\left(f(0), f^{\prime}(0) v\right)=\kappa_{\mathcal{E}(p)}(0, v)$ on $V$.

Proof. By Theorem 3 and its proof, we may assume that $f$ is a linear automorphism of $\mathbb{C}^{n}$ and there exists an open set $U_{1}$ in $\mathcal{E}(p)$ such that on $U_{1}$, the functions $z_{1}, \ldots, z_{n}, f_{1}, \ldots, f_{n}$ do not vanish and $h(f(x))=h(x)$. Then there exists an open connected set $U_{2}$ in $\mathbb{C}^{n}$ such that:

1) $U_{2} \cap \partial \mathcal{E}(p) \neq \emptyset$

2) the mapping $g=\left(z_{1}^{p_{1}}, \ldots, z_{n}^{p_{n}}\right)$ is well-defined and 1-1 on $U_{2}$ and $f\left(U_{2}\right)$,

3) $f\left(U_{2} \cap \partial \mathcal{E}(p)\right) \subset \partial \mathcal{E}(p)$.

Then the map $F=g \circ f \circ g^{-1}$ is holomorphic and 1-1 on $g\left(U_{2}\right)$ and $F\left(g\left(U_{2}\right) \cap \partial \mathbb{B}^{n}\right) \subset \partial \mathbb{B}^{n}$. By the proof of Theorem 1.1 and Corollary 1.2 of Dini and Primicerio [1], $f$ is a linear automorphism of $\mathcal{E}(p)$.

COROLlaRY 1. Let $f$ be a holomorphic map from $D=\left\{\left(z_{1}, \ldots, z_{n}\right) \mid\right.$ $\left.\|z\|_{q}^{q}=\sum_{j=1}^{n}\left|z_{j}\right|^{q}<1\right\}(q \geq 1)$ to itself such that $f(0)=0$. Let $M$ be a connected complex submanifold of dimension $n-1$ of an open subset $U$ of $D$ such that $a+T_{a}(M)$ does not contain the origin for some a in $M$. Let $V$ be a connected open subset of $T_{0}(D)$. If one of the following conditions is satisfied, then $f$ is a linear automorphism of $D$. 
$\left(\mathrm{H}_{1}^{\prime \prime}\right)\|f(x)\|_{q}=\|x\|_{q}$ on $M$.

$\left(\mathrm{H}_{2}^{\prime \prime}\right) \widetilde{k}_{D}(f(0), f(x))=\widetilde{k}_{D}(0, x)$ on $M$.

$\left(\mathrm{H}_{3}^{\prime \prime}\right) \kappa_{D}\left(f(0), f^{\prime}(0) v\right)=\kappa_{D}(0, v)$ on $V$.

EXAMPLE 1. Let $f(z)=\left(z_{1}, \ldots, z_{n-1}, z_{n}^{2}\right)$. Then $f$ maps $\mathcal{E}(p)$ into itself and $f(0)=0$.

(i) Let $M=\left\{z_{n}=0\right\}$. We have $h(f(z))=h(z)$ on $M$. Since $f$ is not linear, the condition that $a+T_{a}(M)$ does not contain the origin cannot be omitted.

(ii) For $k \geq 2$, let $M_{n-k}=\left\{z_{n-k+1}=b, z_{n-k+2}=\ldots=z_{n}=0\right\}$, where $b \neq 0$. The complex dimension of $M_{n-k}$ is $n-k$, and for any $a \in M_{n-k}$, $a+T_{a}(M)$ does not contain the origin. Since $h(f(z))=h(z)$ on $M_{n-k}$ and $f$ is not linear, the condition that the complex dimension of $M$ is $n-1$ cannot be omitted.

\section{References}

[1] G. Dini and A. S. Primicerio, Proper holomorphic mappings between generalized pseudoellipsoids, Ann. Mat. Pura Appl. (4) 158 (1991), 219-229.

[2] H. Hamada, A Schwarz lemma in several complex variables, in: Proc. Third International Colloquium on Finite or Infinite Dimensional Complex Analysis (Seoul, 1995), Kyushu Univ. Co-op., Fukuoka, Japan, 1995, 105-110.

[3] M. Jarnicki and P. Pflug, Invariant Distances and Metrics in Complex Analysis, de Gruyter, Berlin, 1993.

[4] L. Lempert, Holomorphic retracts and intrinsic metrics in convex domains, Anal. Math. 8 (1982), 257-261.

[5] - Intrinsic distances and holomorphic retracts, in: Complex Analysis and Applications '81, Bulgar. Acad. Sci., Sophia, 1984, 341-364.

[6] H. L. Royden and P. M. Wong, Carathéodory and Kobayashi metrics on convex domains, preprint.

[7] J. P. Vigué, Un lemme de Schwarz pour les domaines bornés symétriques irréductibles et certains domaines bornés strictement convexes, Indiana Univ. Math. J. 40 (1991), 293-304.

[8] - Le lemme de Schwarz et la caractérisation des automorphismes analytiques, Astérisque 217 (1993), 241-249.

Faculty of Engineering

Kyushu Kyoritsu University

Jiyugaoka, Yahatanishi-ku

Kitakyushu 807, Japan

E-mail: hamada@kyukyo-u.ac.jp 